Research Article

\title{
Prediction of Vibrational Behavior of Grid-Stiffened Cylindrical Shells
}

\author{
G. H. Rahimi, ${ }^{1}$ M. Hemmatnezhad, ${ }^{1}$ and R. Ansari ${ }^{2}$ \\ ${ }^{1}$ Department of Mechanical Engineering, Tarbiat Modares University, P.O. Box 14115-143 Tehran, Iran \\ ${ }^{2}$ Department of Mechanical Engineering, University of Guilan, P.O. Box 3756, Rasht, Iran
}

Correspondence should be addressed to G. H. Rahimi; rahimi_gh@modares.ac.ir

Received 26 January 2014; Revised 18 April 2014; Accepted 14 May 2014; Published 10 July 2014

Academic Editor: Joseph CS Lai

Copyright (C) 2014 G. H. Rahimi et al. This is an open access article distributed under the Creative Commons Attribution License, which permits unrestricted use, distribution, and reproduction in any medium, provided the original work is properly cited.

\begin{abstract}
A unified analytical approach is applied to investigate the vibrational behavior of grid-stiffened cylindrical shells with different boundary conditions. A smeared method is employed to superimpose the stiffness contribution of the stiffeners with those of shell in order to obtain the equivalent stiffness parameters of the whole panel. Theoretical formulation is established based on Sanders' thin shell theory. The modal forms are assumed to have the axial dependency in the form of Fourier series whose derivatives are legitimized using Stoke's transformation. A 3D finite element model is also built using ABAQUS software which takes into consideration the exact geometric configuration of the stiffeners and the shell. The achievements from the two types of analyses are compared with each other and good agreement has been obtained. The Influences of variations in shell geometrical parameters, boundary condition, and changes in the cross stiffeners angle on the natural frequencies are studied. The results obtained are novel and can be used as a benchmark for further studies. The simplicity and the capability of the present method are also discussed.
\end{abstract}

\section{Introduction}

Investigating the vibrational behavior of cylindrical shells (e.g., frequencies, mode shapes, and modal forces) is of high importance in structural dynamics. Cylindrical shells due to their high strength as well as light weight have gained many applications in the aviation and aerospace industries (e.g., launch vehicles, reentry vehicles, aircraft fuselages, spacecrafts, etc.) in particular. Great number of researches have been emerged rapidly in the past decades concerning with the vibrational analysis of composite cylindrical shells. An excellent review of researches in this area was collected by Leissa [1]. There are also some good reviews on vibration of composite shells using experimental $[2,3]$ and analytical methods [4-9] and numerical techniques [1014]. Recently, Hemmatnezhad et al. [15] investigated the vibrational behavior of composite cylindrical shells using a unified analytical approach and based on different shell theories.
Grid-stiffened cylinders are cylinders reinforced with different types of stiffening structures either on the inner, outer, or both sides of the shell. These stiffeners significantly increase the load resistance of a cylinder without much increase in weight. The selection of stiffener configuration depends on several factors such as the loading condition, cost, and other factors. The promising future of stiffened cylinders, with reinforcing grids or ribs, has led to a wide range of research work [16-23].

The number of publications performed on the mechanical behavior of composite cylinders with cross stiffeners is scarce and most of the relative researches are associated with stiffened cylinders with longitudinal and circumferential stiffeners. Kidane et al. [24, 25] derived the buckling loads of a generally cross and horizontal grid-stiffened composite cylinder by developing a smeared method for determination of the equivalent stiffness parameters of a grid-stiffened composite cylindrical shell. The stiffness contribution of the stiffeners was superimposed with those of the shell to obtain 
the equivalent stiffness parameters of the whole panel. Then, energy method was implemented to obtain the buckling load for a particular stiffener configuration. Buckling test was also performed on a stiffened composite cylinder and the experimental results compared with analytical ones and satisfactory agreement has been achieved. Yazdani et al. performed experimental investigations on the buckling behavior of composite cylindrical shells with cross stiffeners [26]. They also studied the effects of helical ribs' number and changes in grid types on the buckling load of these structures [27, 28]. Recently, Rahimi and his coassociates studied the effect of stiffener cross-section profile on the buckling strength of composite stiffened cylindrical shells by implementing the finite element method [29]. In very recent years, Shi et al. [30] presented the initial buckling and postbuckling responses of axially loaded grid-stiffened composite cylindrical shells with reinforced rectangular or circular cutouts using finite element analysis. Afterwards they extended their previous study and obtained the critical buckling loads of grid-stiffened composite conical cylindrical shells using the minimum potential energy principle [31].

The number of publications concerned with the free vibration analysis of stiffened cylindrical shells with helical ribs is very scarce. To the best of the authors' knowledge, the very recent research on this subject is related to Hemmatnezhad et al. [32]. They implemented an exact analytical approach (as in [15]) investigating the vibrational behavior of grid-stiffened composite cylindrical shells considering the flexural behavior of the ribs. A smeared method was employed to superimpose the stiffness contribution of the stiffeners with those of shell in order to obtain the equivalent stiffness parameters of the whole panel. The stiffeners were modeled as a beam and considered to support shear loads and bending moments further to the axial loads. Therefore, the corresponding stiffness terms are taken into consideration while obtaining the stiffness matrices due to the stiffeners. Theoretical formulations were based on first order shear deformation shell theory which included the effects of transverse shear deformation and rotary inertia.

The aim of the present investigation is to propose a simpler but still accurate method capable of predicting the natural frequencies of stiffened cylindrical shells. A calculation of overall response of simply supported and fully clamped isotropic and composite cylindrical shells with cross stiffeners is presented using an exact analytical approach. Theoretical formulations are based upon Sanders' thin shell theory. A smeared method is employed to superimpose the stiffness contribution of the stiffeners with those of shell in order to obtain the equivalent stiffness parameters of the whole panel $[24,25]$. As stated in $[24,25]$, it is assumed that the transverse modulus of the unidirectional stiffeners is much lower than the longitudinal one and therefore the stiffeners are assumed to behave like a truss element and support the axial load only. A 3D finite element model is also built using ABAQUS software which takes into consideration the exact geometric configuration of the stiffeners and the shell. The influences of the presence of the stiffeners, variations in shell geometrical parameters, and changes in the cross stiffeners angle on the shell frequencies are studied. The results obtained are novel and can be used as a benchmark for further studies.

\section{Equivalent Stiffness}

Consider a cylindrical shell reinforced with a lozenge-type stiffener structure as shown in Figure 1. First of all it is required to determine the equivalent stiffness parameters of the overall structure in order to calculate the vibration frequencies of a composite cylinder with inner stiffening structure. The analytical tool employed for this, so-called the smeared stiffener approach, uses a mathematical model to smear the stiffeners into an equivalent laminate and determine the equivalent stiffness of the laminate (for further details the reader is referred to $[24,25])$.

The relationships between boundary forces and strains for a cylindrical shell are given as

$$
\begin{aligned}
{\left[\begin{array}{l}
N \\
M
\end{array}\right] } & =\left[\begin{array}{c}
V_{s} N^{s}+V_{\mathrm{sh}} N^{\mathrm{sh}} \\
V_{s} M^{s}+V_{\mathrm{sh}} M^{\mathrm{sh}}
\end{array}\right] \\
& =\left[\frac{V_{s} A^{s}+V_{\mathrm{sh}} A^{\mathrm{sh}} \mid V_{s} B^{s}+V_{\mathrm{sh}} B^{\mathrm{sh}}}{V_{s} B^{s}+V_{\mathrm{sh}} B^{\mathrm{sh}} \mid V_{s} D^{s}+V_{\mathrm{sh}} D^{\mathrm{sh}}}\right]\left[\begin{array}{c}
\varepsilon^{\mathrm{o}} \\
\kappa
\end{array}\right],
\end{aligned}
$$

where the $s$ and sh superscripts stand for the stiffener and shell, respectively. In the above equation, $V_{s}$ and $V_{\text {sh }}$ are the volume fractions of the stiffeners and shell, respectively, $N^{s}, M^{s}$ and $N^{\text {sh }}, M^{\text {sh }}$ represent the force and moment contributions of the stiffeners and shell, respectively. $A, B$, and $D$ indicate the extensional, coupling, and bending stiffness matrices, respectively. Therefore, the equivalent stiffness coefficients of the whole panel can be obtained from

$$
\begin{aligned}
& {[A]=V_{s}[A]^{s}+V_{\mathrm{sh}}[A]^{\mathrm{sh}},} \\
& {[B]=V_{s}[B]^{s}+V_{\mathrm{sh}}[B]^{\mathrm{sh}},} \\
& {[D]=V_{s}[D]^{s}+V_{\mathrm{sh}}[D]^{\mathrm{sh}} .}
\end{aligned}
$$

The resultant forces and moments due to the shell in terms of the strain components of the midplane surface of the shell can be written as

$$
\left\{\begin{array}{l}
N_{x}^{\text {sh }} \\
N_{\theta}^{\text {sh }} \\
N_{x \theta}^{\text {sh }} \\
M_{x}^{\text {sh }} \\
M_{\theta}^{\text {sh }} \\
M_{x \theta}^{\text {sh }}
\end{array}\right\}=\left[\begin{array}{cccccc}
A_{11} & A_{12} & 0 & B_{11} & B_{12} & 0 \\
A_{12} & A_{22} & 0 & B_{12} & B_{22} & 0 \\
0 & 0 & A_{66} & 0 & 0 & B_{66} \\
B_{11} & B_{12} & 0 & D_{11} & D_{12} & 0 \\
B_{12} & B_{22} & 0 & D_{12} & D_{22} & 0 \\
0 & 0 & B_{66} & 0 & 0 & D_{66}
\end{array}\right]^{\text {sh }}\left\{\begin{array}{c}
\varepsilon_{x}^{\mathrm{o}} \\
\varepsilon_{\theta}^{\mathrm{o}} \\
\gamma_{x \theta}^{\mathrm{o}} \\
K_{x} \\
K_{\theta} \\
K_{x \theta}
\end{array}\right\}
$$



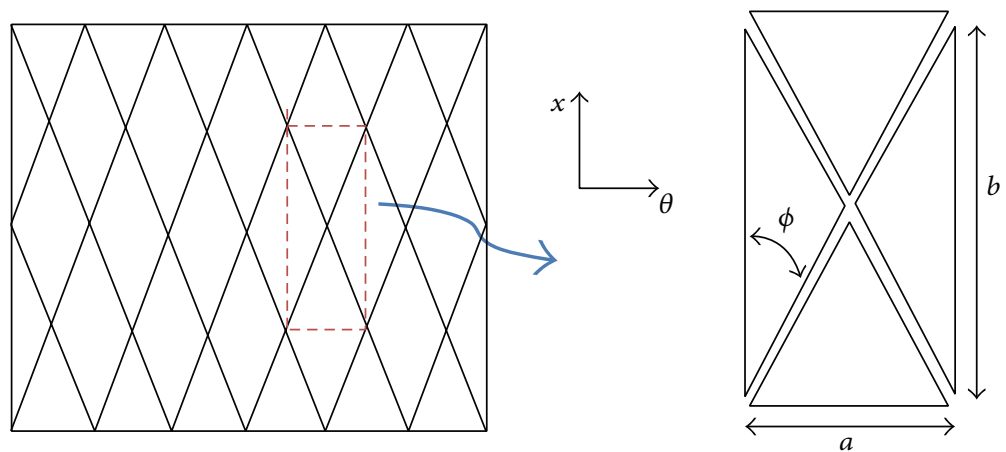

FIGURE 1: Unit cell and coordinate system for a stiffened cylindrical shell.

while those due to the stiffeners are as follows:

$$
\begin{aligned}
& {\left[\begin{array}{c}
N_{x}^{s} \\
N_{\theta}^{s} \\
N_{x \theta}^{s} \\
M_{x}^{s} \\
M_{\theta}^{s} \\
M_{x \theta}^{s}
\end{array}\right]} \\
& =A E_{1}\left[\begin{array}{cccccc}
\frac{2 c^{3}}{a} & \frac{2 s^{2} c}{a} & 0 & \frac{c^{3} t}{a} & \frac{s^{2} c t}{a} & 0 \\
\frac{2 s c^{2}}{b} & \frac{2 s^{3}}{b} & 0 & \frac{s c^{2} t}{b} & \frac{2 s^{3} t}{2 b} & 0 \\
\frac{c^{3} t}{a} & \frac{s^{2} c t}{a} & 0 & \frac{c^{3} t^{2}}{2 a} & \frac{s^{2} c t^{2}}{2 a} & 0 \\
\frac{s c^{2} t}{b} & \frac{2 s^{3} t}{2 b} & 0 & \frac{s c^{2} t^{2}}{2 b} & \frac{2 s^{3} t^{2}}{4 b} & 0 \\
0 & 0 & \frac{s c^{2} t}{b} & 0 & 0 & \frac{s c^{2} t^{2}}{2 b}
\end{array}\right]\left[\begin{array}{c}
\varepsilon_{x}^{0} \\
\varepsilon_{\theta}^{0} \\
\varepsilon_{\theta x}^{0} \\
\kappa_{x} \\
\kappa_{\theta} \\
\kappa_{x \theta}
\end{array}\right],
\end{aligned}
$$

where $c=\cos \phi, s=\sin \phi$, and $\phi$ is the stiffener orientation angle. $a, b$, and $t$ are the cross stiffeners spacing, unit cell height, and shell thickness, respectively. In (3) $A_{i j}, B_{i j}$, and $D_{i j}$ are given by relation as follows:

$$
\left|A_{i j}, B_{i j}, D_{i j}\right|^{\text {sh }}=\sum_{k=1}^{p} \int_{h_{k-1}}^{h_{k}} Q_{i j}^{(k)}\left|1, z, z^{2}\right| d z,
$$

where $Q_{i j}^{(k)}$ are known as the elastic moduli of the $k$ th layer. $\varepsilon$ and $\kappa$ are the strains and curvatures defined as as follows based on Sanders' thin shell theory:

$$
\begin{gathered}
\varepsilon_{x}^{0}=u_{, x}, \quad \varepsilon_{\theta}^{0}=\frac{1}{R}\left(v_{, \theta}\right), \quad \varepsilon_{\theta x}^{0}=v_{, x}+\frac{1}{R} u_{, \theta}, \\
\kappa_{x}=-w_{, x x}, \quad \kappa_{\theta}=-\frac{1}{R^{2}}\left(w_{, \theta \theta}-v_{, \theta}\right), \\
k_{x \theta}=\frac{-2}{R}\left(w_{, x \theta}-\frac{3}{4} v_{, x}+\frac{1}{4 R} u_{, \theta}\right) .
\end{gathered}
$$

\section{Equations of Motion}

A multilayered circular cylindrical shell with lozenge-type stiffener structure is chosen for the present analysis. The geometrical parameters associated with the shell are described by $R$, the radius of the shell's middle surface; $L$, length of the shell; and $t$, thickness of the shell. The following shell equations according to Sanders [33], in terms of axial, $x$, and circumferential, $\theta$, coordinates, are used:

$$
\begin{gathered}
R N_{x, x}+N_{x \theta, \theta}=R I_{1} \ddot{u} \\
R N_{x \theta, x}+N_{\theta \theta, \theta}+\frac{1}{R} M_{\theta \theta, \theta}+M_{x \theta, x}=R I_{1} \ddot{v} \\
R M_{x, x x}+2 M_{x \theta, x \theta}+\frac{1}{R} M_{\theta, \theta \theta}-N_{\theta}=R I_{1} \ddot{w},
\end{gathered}
$$

where $u, v$, and $w$ are the axial, tangential, and radial displacements, respectively. A comma before a subscript indicates the differentiation with respect to that subscript and dot denotes the time derivative. In (7), $I_{1}$ is the inertia term for composite shell which can be obtained as

$$
I_{1}=\sum_{k=1}^{p} \int_{h_{k-1}}^{h_{k}} \rho^{(k)} d z
$$

in which $\rho^{(k)}$ is the density of the $k$ th layer.

\section{Analytical Procedure}

4.1. Theoretical Considerations. The basic equations presented in the previous sections will now be used to study the vibration characteristics of thin circular cylindrical shells. The general analytical procedure presented here is for evaluating the natural frequencies of multilayered orthotropic circular cylindrical shells with arbitrary boundary conditions. The case concerning single-layered and also isotropic shells can come out as a particular case of the analysis. 
4.2. Field Equations. Utilizing (1) and (6), (7) can be expressed in terms of displacement field and its corresponding derivatives as follows:

$$
\begin{aligned}
& R\left[A_{11} u_{, x x}+\frac{A_{12}}{R}\left(v_{, x \theta}+w_{, x}\right)\right. \\
& \left.-B_{11} w_{, x x x}+\frac{B_{12}}{R^{2}}\left(v_{, x \theta}-w_{, x \theta \theta}\right)\right] \\
& +A_{66}\left(v_{, x \theta}+\frac{1}{R} u_{, \theta \theta}\right) \\
& +\frac{B_{66}}{2 R}\left(v_{, x \theta}-2 w_{, x \theta \theta}\right)=R I_{1} \ddot{u} \\
& R\left[A_{66}\left(v_{, x x}+\frac{1}{R} u_{, \theta x}\right)\right. \\
& \left.+\frac{B_{66}}{2 R}\left(v_{, x x}-2 w_{, x x \theta}\right)\right] \\
& +A_{12} u_{, x \theta}+\frac{A_{22}}{R}\left(v_{, \theta \theta}+w_{, \theta}\right) \\
& -B_{12} w_{, x x \theta}+\frac{B_{22}}{R^{2}}\left(v_{, \theta \theta}-w_{, \theta \theta \theta}\right) \\
& +\frac{1}{R}\left[B_{12} u_{, x \theta}+\frac{B_{22}}{R}\left(v_{, \theta \theta}+w_{, \theta}\right)\right. \\
& \left.-D_{12} w_{, x x \theta}+\frac{D_{22}}{R^{2}}\left(v_{, \theta \theta}-w_{, \theta \theta \theta}\right)\right] \\
& +B_{66}\left(v_{, x x}+\frac{1}{R} u_{, x \theta}\right) \\
& +\frac{D_{66}}{2 R}\left(v_{, x x}-2 w_{, x x \theta}\right)=R I_{1} \ddot{v} \\
& R\left[B_{11} u_{, x x x}+\frac{B_{12}}{R}\left(v_{, x x \theta}+w_{, x x}\right)\right. \\
& \left.-D_{11} w_{, x x x x}+\frac{D_{12}}{R^{2}}\left(v_{, x x \theta}-w_{, x x \theta \theta}\right)\right] \\
& +2 B_{66}\left(v_{, x x \theta}+\frac{1}{R} \times u_{, x \theta \theta}\right) \\
& +\frac{D_{66}}{R}\left(v_{, x x \theta}-2 w_{, x x \theta \theta}\right) \\
& +\frac{1}{R}\left[B_{12} u_{, x \theta \theta}+\frac{B_{22}}{R}\left(v_{, \theta \theta \theta}+w_{, \theta \theta}\right)\right. \\
& \left.-D_{12} w_{, x x \theta \theta}-\frac{D_{22}}{R^{2}} \times\left(w_{, \theta \theta \theta \theta}-v_{, \theta \theta \theta}\right)\right] \\
& -\left[A_{12} u_{, x}+\frac{A_{22}}{R}\left(v_{, \theta}+w\right)\right. \\
& \left.-B_{12} w_{, x x}+\frac{B_{22}}{R^{2}}\left(v_{, \theta}-w_{, \theta \theta}\right)\right]=R I_{1} \ddot{w} .
\end{aligned}
$$

is chosen as a base problem for the set of displacement functions given in (11). None of the eight boundary conditions given by (13) are satisfied by the CSS set on a term-by-term basis. Therefore, Stoke's transformation is used to enforce constraints to satisfy the boundary conditions [15]. Since sine series give zero values at the ends, the following affected terms are specified while differentiating:

$$
\begin{array}{cc}
\Psi_{v}(0)=v_{0}, & \Psi_{v}(L)=v_{L}, \\
\Psi_{w}(0)=w_{0}, & \Psi_{w}(L)=w_{L}, \\
\Psi_{\psi_{\theta}}(0)=\psi_{\theta}^{0}, & \Psi_{\psi_{\theta}}(L)=\psi_{\theta}^{L} .
\end{array}
$$


4.4. General Formulations. Substitution of the set of displacement functions and their derivatives into (9a)-(9c) leads to an explicit relation for $A_{0 n}$ and a matrix equation in which $A_{m n}$, $B_{m n}$, and $C_{m n}$ are coupled together as

$$
\begin{gathered}
\left\{\left[\begin{array}{ccc}
K_{11} & K_{12} & K_{13} \\
& K_{22} & K_{23} \\
\text { Symm. } & & K_{33}
\end{array}\right]-\left[\begin{array}{ccc}
I_{1} & 0 & 0 \\
0 & I_{1} & 0 \\
0 & 0 & I_{1}
\end{array}\right] \omega^{2}\right\}\left[\begin{array}{l}
A_{m n} \\
B_{m n} \\
C_{m n}
\end{array}\right] \\
=\left[\begin{array}{l}
F_{1} \\
F_{2} \\
F_{3}
\end{array}\right], \\
\left(K_{01}-I_{1} \omega^{2}\right) A_{0 n}=F_{01},
\end{gathered}
$$

where

$$
\begin{aligned}
& K_{11}=R A_{11}\left(\frac{m \pi}{L}\right)^{2}+A_{66} \frac{n^{2}}{R}, \\
& K_{12}=-\left(A_{12}+A_{66}+\frac{B_{12}}{R}+\frac{B_{66}}{2 R}\right)\left(\frac{m n \pi}{L}\right) \text {, } \\
& K_{13}=-A_{12}\left(\frac{m \pi}{L}\right)-R B_{11}\left(\frac{m \pi}{L}\right)^{3}+\left(B_{12}-B_{66}\right)\left(\frac{m \pi n^{2}}{R L}\right) \text {, } \\
& K_{22}=\left(R A_{66}+\frac{3 B_{66}}{2}+\frac{D_{66}}{2 R}\right)\left(\frac{m \pi}{L}\right)^{2} \\
& +\left(R A_{22}+2 B_{22}+\frac{D_{22}}{R}\right)\left(\frac{n}{R}\right)^{2}, \\
& K_{33}=\left(\frac{m \pi}{L}\right)^{2}\left(2 B_{12}+R D_{11}\left(\frac{m \pi}{L}\right)^{2}+2 \frac{D_{12}+D_{66}}{R} n^{2}\right) \\
& +2 B_{22}\left(\frac{n}{R}\right)^{2}+\frac{A_{22}}{R}+D_{22} \frac{n^{4}}{R^{3}} \\
& K_{01}=A_{66} \frac{n^{2}}{R} \\
& F_{1}=f_{1}\left(\bar{u}_{0}+\bar{u}_{L}(-1)^{m}\right)+f_{2}\left(v_{0}+v_{L}(-1)^{m}\right) \\
& +f_{3}\left(w_{0}+w_{L}(-1)^{m}\right)+f_{4}\left(\overline{\bar{w}}_{0}+\overline{\bar{w}}_{L}(-1)^{m}\right), \\
& F_{2}=f_{5}\left(v_{0}+v_{L}(-1)^{m}\right)+f_{6}\left(w_{0}+w_{L}(-1)^{m}\right) \text {, } \\
& F_{3}=f_{7}\left(v_{0}+v_{L}(-1)^{m}\right)+f_{8}\left(w_{0}+w_{L}(-1)^{m}\right) \\
& +f_{9}\left(\overline{\bar{w}}_{0}+\overline{\bar{w}}_{L}(-1)^{m}\right) \\
& F_{01}=f_{10}\left(\bar{u}_{0}+\bar{u}_{L}(-1)^{m}\right)+f_{11}\left(v_{0}+v_{L}(-1)^{m}\right) \\
& +f_{12}\left(w_{0}+w_{L}(-1)^{m}\right)+f_{13}\left(\overline{\bar{w}}_{0}+\overline{\bar{w}}_{L}(-1)^{m}\right) .
\end{aligned}
$$

The four quantities, $\bar{u}_{0}, \bar{u}_{L}, \overline{\bar{w}}_{0}$, and $\overline{\bar{w}}_{L}$, are associated with the unspecified end forces $N_{x}$ and moments $M_{x}$ at the shell ends. Therefore, using (15), $A_{m n}, B_{m n}, C_{m n}$, and $A_{0 n}$ can now be expressed explicitly in terms of the eight unspecified boundary values $N_{x}^{0}, N_{x}^{L}, M_{x}^{0}, M_{x}^{L}, v_{0}, v_{L}, w_{0}$, and $w_{L}$. As mentioned before, none of the eight boundary conditions of "FSNT" shells as given by (13) are satisfied by the assumed CSS modal displacement forms. Hence, one must enforce these boundary conditions which are both geometrical and natural types. The geometric boundary conditions that must be imposed are related to $u$ and $\partial w / \partial x$, while those of natural type are associated with $N_{x \theta}=0$, and $\left(\partial M_{x} / \partial x\right)+(2 / R)\left(\partial M_{x \theta} / \partial \theta\right)=0$, at both ends. Finally, the eight constraint conditions, due to the geometric and natural boundary conditions, lead to the following homogeneous matrix equation:

$$
\begin{array}{r}
{\left[e_{i j}\right]\left[\begin{array}{llllllll}
N_{x}^{0} & N_{x}^{L} & M_{x}^{0} & M_{x}^{L} & v_{0} & v_{L} & w_{0} & w_{L}
\end{array}\right]^{T}=[0],} \\
(i, j=1,2, \ldots, 8) .
\end{array}
$$

For a nontrivial solution of (17), the determinant of the coefficient matrix must vanish, as

$$
\left|e_{i j}\right|=0, \quad(i, j=1,2, \ldots, 8)
$$

resulting in a characteristic equation whose eigenvalues are the natural frequencies of the stiffened shell. The corresponding eigenvectors also determine the mode shapes. To derive the appropriate characteristic equation of a specified boundary condition, its associated end conditions must be imposed. This can be either performed by appropriately tailoring the general determinant of (18). The procedure regarding this is discussed in detail in [15].

As a sample, since each of the boundary conditions of a SNA-SNA shell is satisfied by the CSS set on a term-byterm basis, the frequency equation for this type of boundary condition is directly obtained from the equations of motion as a three-by-three determinant of (15) as

$$
\left|\begin{array}{ccc}
K_{11}-I_{1} \omega^{2} & K_{12} & K_{13} \\
& K_{22}-I_{1} \omega^{2} & K_{23} \\
\text { Symm. } & & K_{33}-I_{1} \omega^{2}
\end{array}\right|=0
$$

whose roots give the natural frequencies of SNA-SNA gridstiffened cylindrical shells.

\section{Results and Discussion}

A 3D model of a grid-stiffened cylinder with three couples of reinforcing ribs whose vibrational behavior is to be investigated in the present work is depicted in Figure 2. The stiffener-cylinder structure is considered to be made of steel with material properties as $E=206 \mathrm{GPa}, \rho=7800 \mathrm{~kg} / \mathrm{m}^{3}$. The geometrical parameters are taken as

$$
\begin{gathered}
L=253.9 \mathrm{~mm}, \quad R=70.5 \mathrm{~mm}, \quad t=1 \mathrm{~mm}, \\
\phi=30^{\circ}, \quad a=\frac{2 \pi R}{3}, \\
b=\frac{a}{\tan (\phi)}, \quad A_{s}=4 * 4 \mathrm{~mm}^{2} .
\end{gathered}
$$

Table 1 illustrates a comparison between the natural frequencies of stiffened and unstiffened SNA-SNA isotropic cylindrical shells for ten values of circumferential mode 


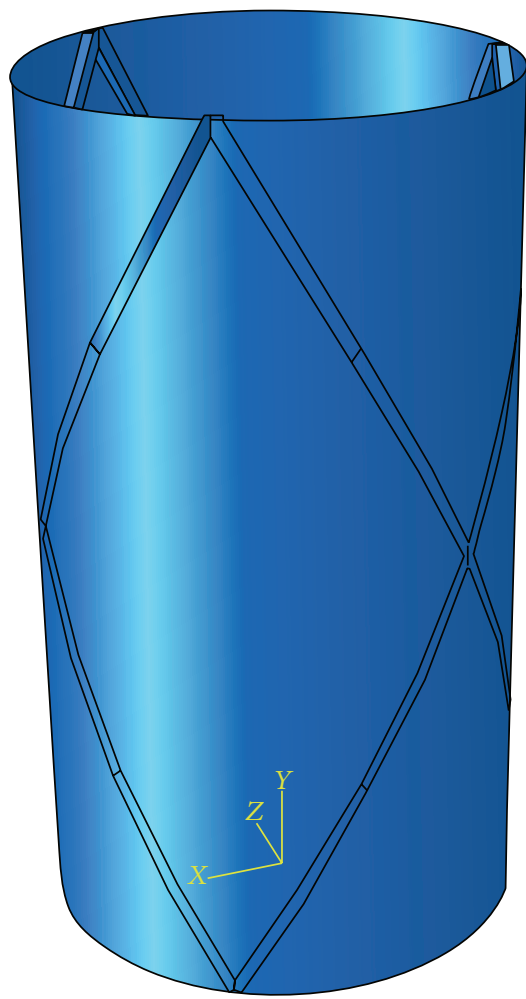

FIGURE 2: A 3D model of the grid-stiffened cylinder used in the present work.

TABLE 1: Comparison of the natural frequencies $(\mathrm{Hz})$ for a SNA-SNA steel cylindrical shell with and without stiffeners.

\begin{tabular}{lcc}
\hline Number & Stiffened shell & Unstiffened shell \\
\hline 1 & 2994.6 & 3599.0 \\
2 & 1367.3 & 1646.1 \\
3 & 778.4 & 943.1 \\
4 & 743.1 & 904.7 \\
5 & 1021.1 & 1241.3 \\
6 & 1448.8 & 1758.7 \\
7 & 1975.6 & 2396.5 \\
8 & 2589.4 & 3139.9 \\
9 & 3286.8 & 3984.9 \\
10 & 4066.9 & 4930.4 \\
\hline
\end{tabular}

numbers. As would be observed, the values of the frequencies for the unstiffened shell of the present model are higher than that of stiffened shell. This is mainly because of the grid structure which results into an increase in the mass and a decrease in the natural frequency as a consequence. Table 2 is a similar one for clamped-clamped (C-C) boundary condition. As expected before, the natural frequencies associated with the C-C shell are higher than that of SNASNA ones. Figure 3 shows the variation of natural frequencies with the circumferential wave number for a stiffened SNASNA cylindrical shell. The results are also compared with those reported via finite element analysis by ABAQUS CAE software. The lozenge-type stiffeners consist of six helical

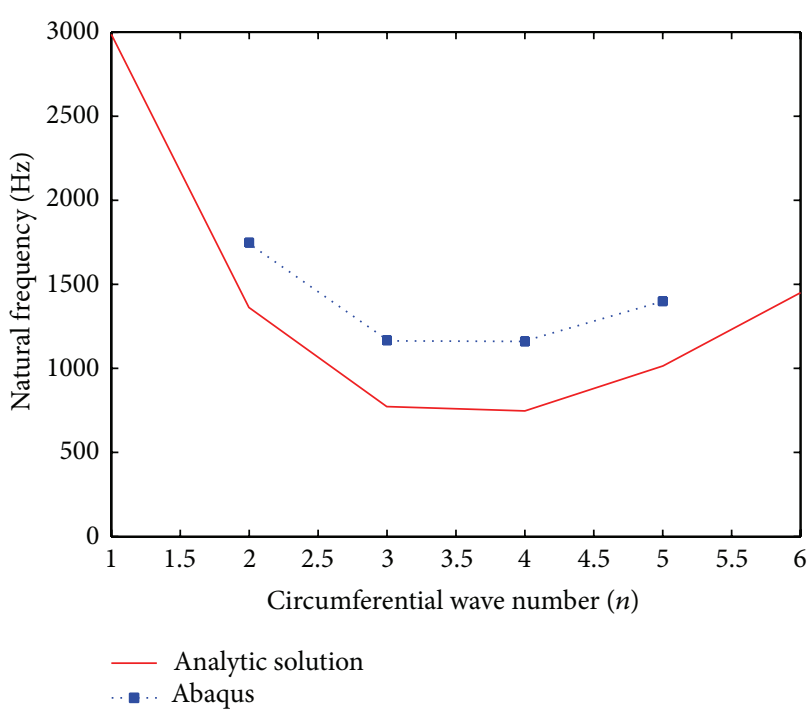

FIGURE 3: Frequency variation for a stiffened SNA-SNA steel cylindrical shell.

TABLE 2: Comparison of the natural frequencies $(\mathrm{Hz})$ for a C-C steel cylindrical shell with and without stiffeners.

\begin{tabular}{lcc}
\hline Number & Stiffened shell & Unstiffened shell \\
\hline 1 & 3472.6 & 4176.1 \\
2 & 2032.9 & 2444.1 \\
3 & 1307.1 & 1573.3 \\
4 & 1042.2 & 1259.2 \\
5 & 1150.0 & 1393.8 \\
6 & 1504.1 & 1824.1 \\
7 & 1975.6 & 2396.5 \\
8 & 2589.4 & 3139.9 \\
9 & 3286.8 & 3984.9 \\
10 & 4066.9 & 4930.4 \\
\hline
\end{tabular}

ribs oriented at $30^{\circ}$ and $-30^{\circ}$ angles with regard to the longitudinal axis of the shell/stiffener structure. The stiffeners are assembled into the shell so that the common nodes on the interfacing areas are merged upon meshing. Therefore, the ribs and shell become a unit structure. The quadratic planar elements with 8 nodes (S8R) and quadratic cubic elements with 20 nodes (C3D20R) have been used to mesh the shell and the ribs, respectively. As can be seen, the trends of the frequency response obtained from the two analyses are in good agreement. The difference between the two methods is mainly due to the simplification assumptions taken in the analytic procedure such as the inconsideration of the bending effect for the stiffeners while calculating the equivalent stiffnesses of the shell-stiffener structure. However, ABAQUS models the shell-stiffener structure with the exact geometric configuration for the stiffeners and therefore considers a stiffer structure rather than that in the analytical analysis. 


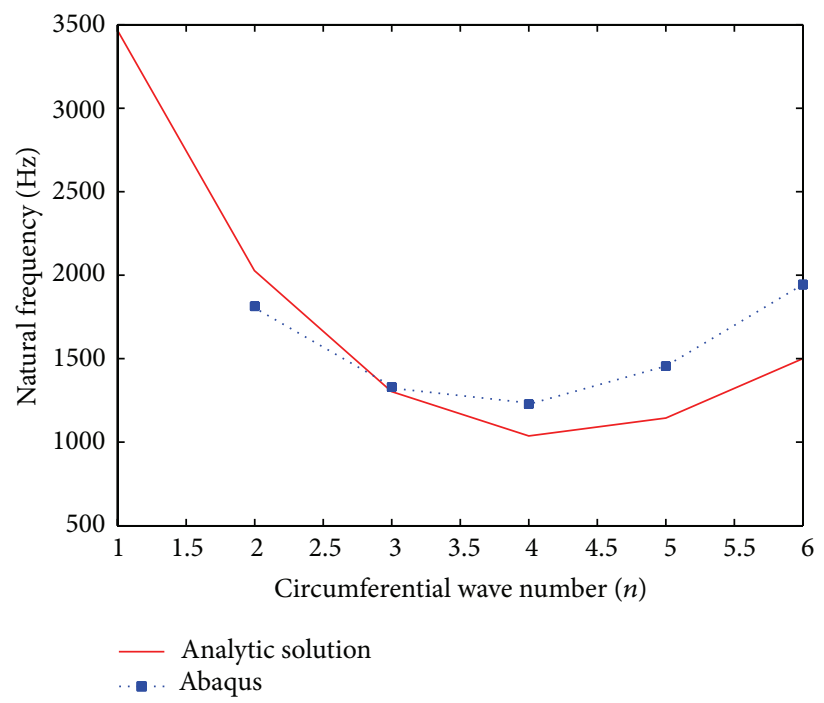

FIGURE 4: Frequency variation for a stiffened C-C steel cylindrical shell.

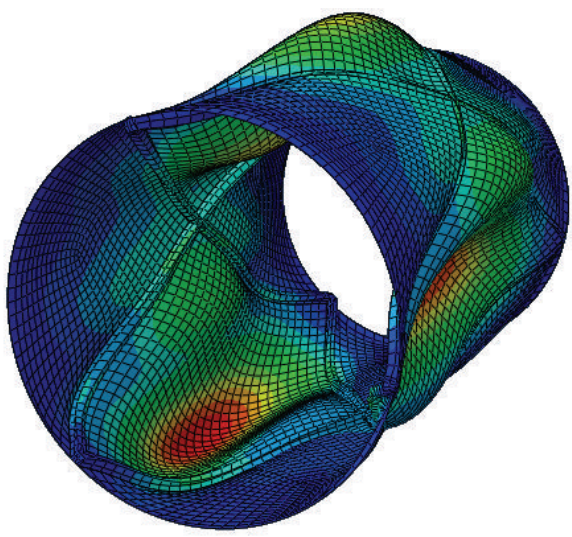

$n=2$

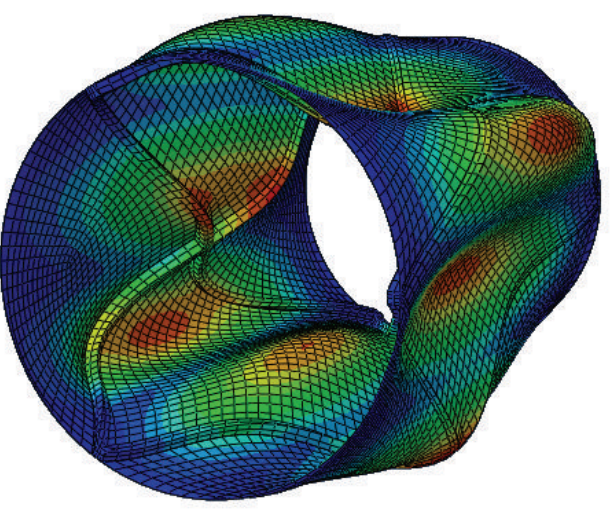

$n=4$

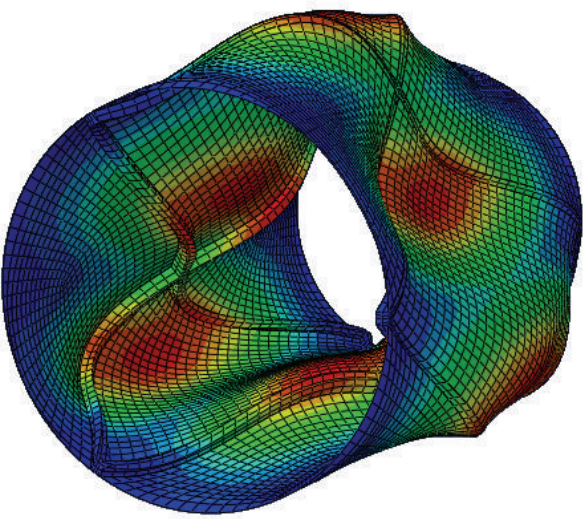

$n=3$

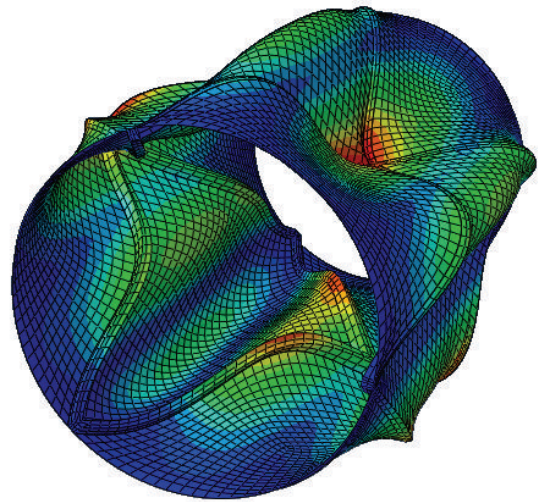

$n=5$

FIGURE 5: Mode shapes associated with SNA-SNA steel stiffened cylindrical shell.

That is why the natural frequencies given by ABAQUS are higher than those reported by the analytical technique. The similar graph for the $\mathrm{C}-\mathrm{C}$ end condition is illustrated in Figure 4. Figures 5 and 6 exhibit the mode shapes associated with SNA-SNA and C-C stiffened cylindrical shells, respectively.
Then, a stiffener-cylinder structure made up of HsGraphite/epoxy is considered for the analysis with material properties listed in Table 3. The cylindrical shell is assumed to be one-layered with fiber angle value of $45^{\circ}$, while in the stiffener structure, fibers are considered to be oriented in the ribs' directions. The variation of the natural frequency with 

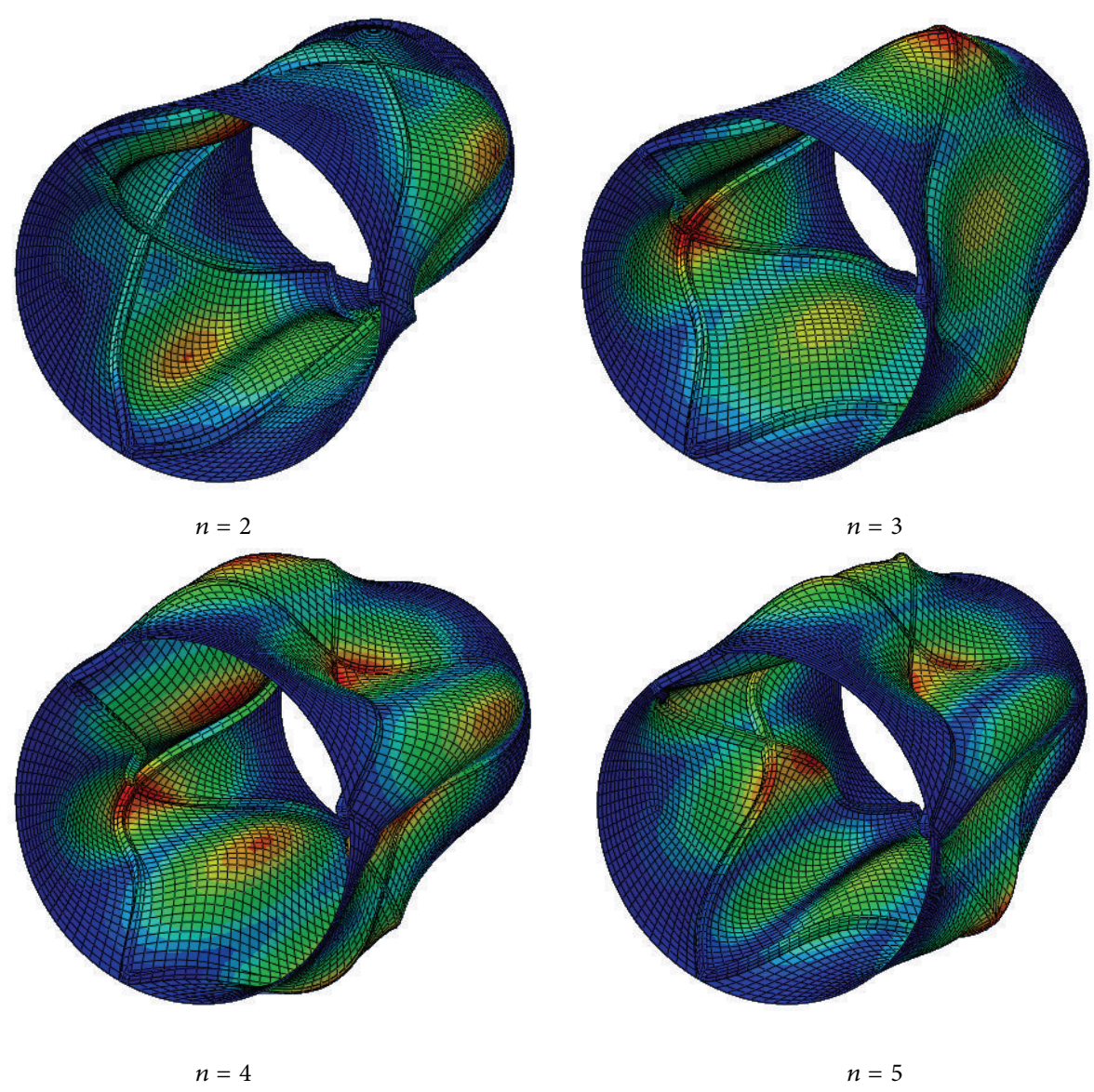

Figure 6: Mode shapes associated with C-C steel stiffened cylindrical shell.

TABle 3: Material properties of Hs-Graphite/epoxy.

\begin{tabular}{lcc}
\hline \multicolumn{3}{c}{ Hs-graphite/epoxy } \\
\hline Young's modulus $(\mathrm{GPa})$ & $E_{11}, E_{22}, E_{33}$ & $181.0,10.34,10.34$ \\
Shear modulus $(\mathrm{Gpa})$ & $G_{12}, G_{13}, G_{23}$ & $7.2,7.2,7.2$ \\
Poisson's ratio & $\nu_{12}, v_{13}, \nu_{23}$ & $0.28,0.28,0.28$ \\
Density $\left(\mathrm{kg} / \mathrm{m}^{3}\right)$ & $\rho$ & 1389.23 \\
\hline
\end{tabular}

the circumferential wave number of a SNA-SNA composite grid-stiffened cylindrical shell is exhibited in Figure 7 for three different values of shell thicknesses. The results are also compared with those of the unstiffened shells. As would be observed, the difference between two curves becomes smaller as the shell thickness increases. This means that, for greater shell thicknesses, the presence of grid structure has no significant effect on the vibrational behavior and the natural frequencies approach to those of the unstiffened structure. Also, it can be pointed out that, for $t=0.2 \mathrm{~mm}$, the natural frequencies of grid-stiffened composite shell surpass those of unstiffened one for $n>3$. This occurs when the stiffness of the structure increases with faster rate than the structure weight. Figure 8 illustrates the variation of the natural frequency with the shell length for first three circumferential modes of vibration and fiber angle of $0^{\circ}$. It can be seen that, with an increment in the shell length, the natural frequencies of both unstiffened and stiffened shells decrease. Also, the influence of shell length variation on the natural frequencies is more significant for $n=1$. Figure 9 plots the variation of natural frequencies with ribs' angle for first three circumferential wave numbers and fiber angle of $0^{\circ}$. It should be said that the cross stiffeners angle has significant effect on the vibration of the stiffened composite cylindrical shell especially for $n=3$.

\section{Conclusion}

A unified exact analysis is employed to investigate the dynamic behavior of stiffened circular cylindrical shells with cross stiffeners. A smeared method is employed to superimpose the stiffness contribution of the stiffeners with those of shell in order to obtain the equivalent stiffness parameters of the whole panel. These equivalent stiffnesses will then be entered into the analytical procedure in order to obtain the natural frequencies of vibration. To validate the correctness of the obtained results, a 3D finite element model is also built using the ABAQUS CAE software. Results given are novel and can be used as a benchmark for further studies. Results clarify that the natural frequencies of stiffened cylinder are often lower than those excluding the effect of stiffeners. However, 


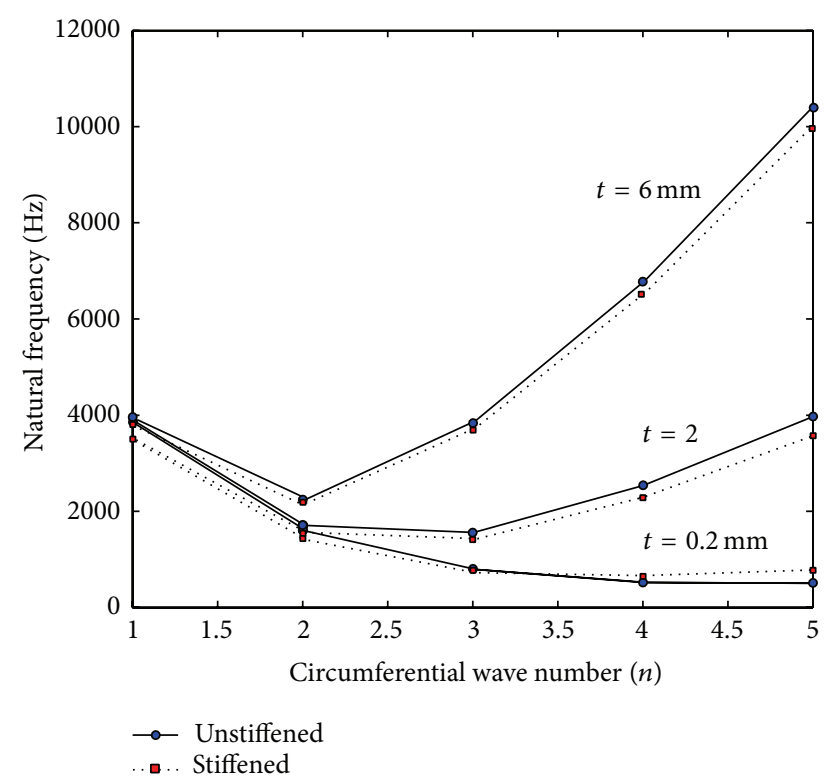

FIGURE 7: Variation of the natural frequency with circumferential wave number for different values of shell thicknesses $(L=$ $253.9 \mathrm{~mm})$.

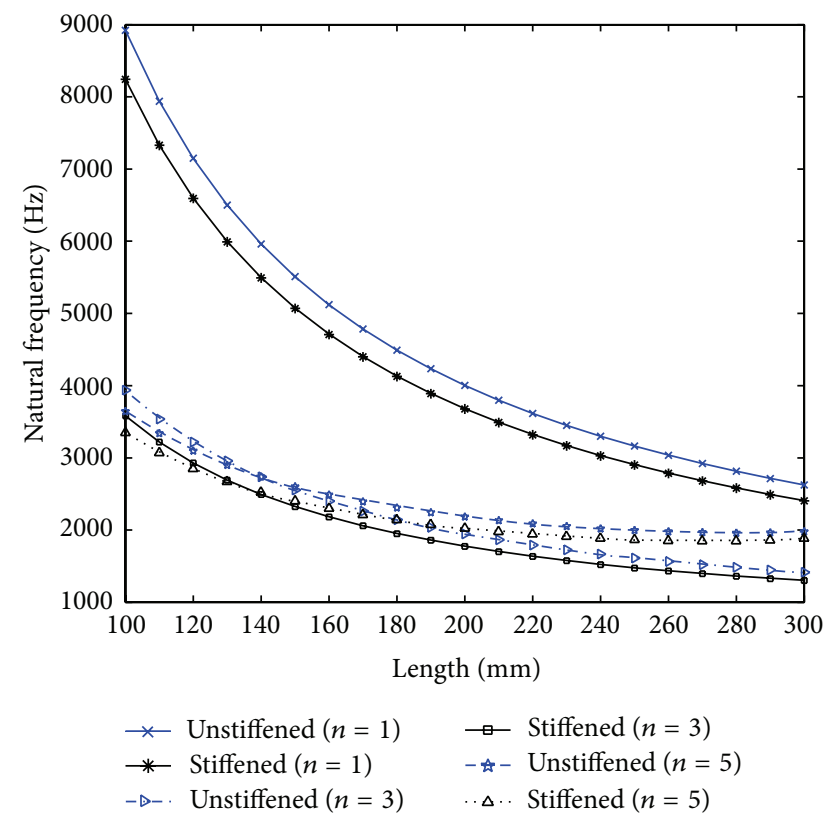

FIGURE 8: Variation of the natural frequency associated with first three circumferential modes with the shell length of a SNA-SNA composite cylindrical shell $(t=2 \mathrm{~mm})$.

in the case of composite stiffened shells it has been seen that the natural frequencies of stiffened cylinder can surpass those of unstiffened counterpart for small shell thicknesses. This occurs when the stiffness of the structure increases with faster rate than the structure weight. Also, the cross stiffeners angle has significant effect on the vibration of the stiffened cylinder especially for higher circumferential modes.

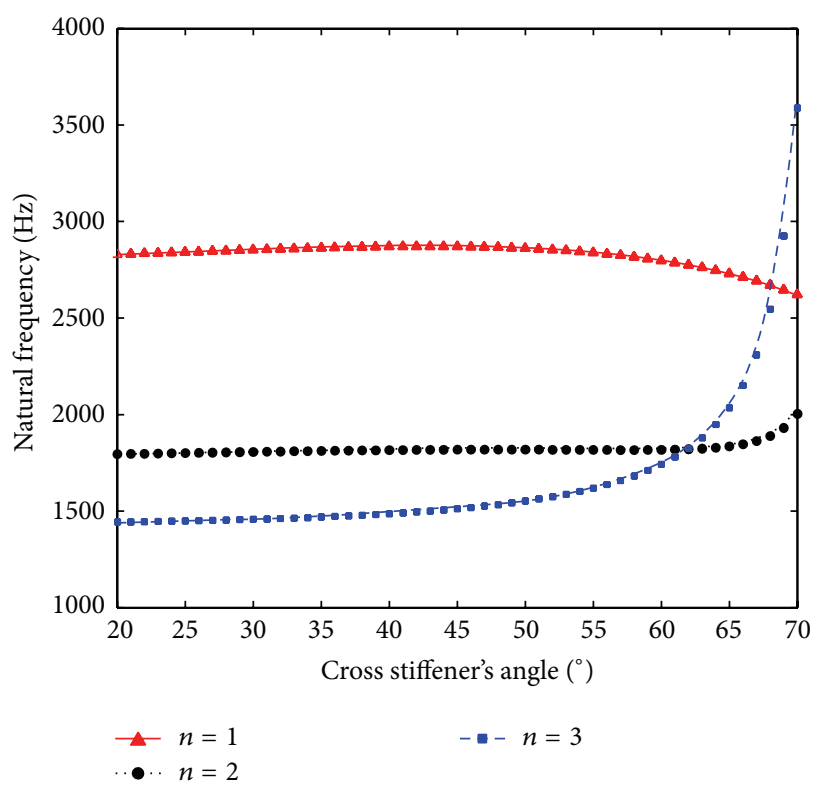

FIGURE 9: Variation of the natural frequency with first three circumferential modes with the cross stiffener's angle of a SNA-SNA composite cylindrical shell $(t=2 \mathrm{~mm})$.

\section{Conflict of Interests}

The authors declare that there is no conflict of interests regarding the publication of this paper.

\section{References}

[1] A. W. Leissa, "Vibration of shells," NASA SP-288 US Govt Printing Office, 1973.

[2] D. M. Egle and F. M. Bray, "An experimental study of free vibration of cylindrical shells with discrete longitudinal stiffening," Final Report GK-1490, School of Aero-Space and Mechanical Engineering, University of Oklahoma, NSF Grant, 1968.

[3] C. B. Sharma, "Calculation of natural frequencies of fixed-free circular cylindrical shells," Journal of Sound \& Vibration, vol. 35, no. 1, pp. 55-76, 1974.

[4] C. B. Sharma and M. Darvizeh, "Free vibration characteristics of laminated, orthographic clamped-free cylindrical shells, developments in mechanics," in Proceedings of the 19th Midwestern Mechanics Conference, vol. 13, Department of Engineering Mechanics, The Ohio State University, Columbus, Ohio, USA, September 1985.

[5] M. Darvizeh, Free vibration characteristics of orthotropic thin circular cylindrical shell [Ph.D. thesis], UMIST, 1985.

[6] C. B. Sharma, M. Darvizeh, and A. Darvizeh, "Free vibration response of multilayered orthotropic fluid-filled circular cylindrical shells," Composite Structures, vol. 34, no. 3, pp. 349-355, 1996.

[7] V. Birman, "Exact solution of axisymmetric problems of laminated cylindrical shells with arbitrary boundary conditionshigher-order theory," Mechanics Research Communications, vol. 19, no. 3, pp. 219-225, 1992.

[8] K. Y. Lam and C. T. Loy, "Influence of boundary conditions and fibre orientation on the natural frequencies of thin orthotropic 
laminated cylindrical shells," Composite Structures, vol. 31, no. 1, pp. 21-30, 1995.

[9] K. Y. Lam and C. T. Loy, "Influence of boundary conditions for a thin laminated rotating cylindrical shell," Composite Structures, vol. 41, no. 3-4, pp. 215-228, 1998.

[10] C. W. Bert and M. Malik, "Differential quadrature: a powerful new technique for analysis of composite structures," Composite Structures, vol. 39, no. 3-4, pp. 179-189, 1997.

[11] C. B. Sharma, M. Darvizeh, and A. Darvizeh, "Free vibration behaviour of helically wound cylindrical shells," Composite Structures, vol. 44, no. 1, pp. 55-62, 1999.

[12] H. Haftchenari, M. Darvizeh, A. Darvizeh, R. Ansari, and C. B. Sharma, "Dynamic analysis of composite cylindrical shells using differential quadrature method (DQM)," Composite Structures, vol. 78, no. 2, pp. 292-298, 2007.

[13] N. Ganesan and R. Kadoli, "Buckling and dynamic analysis of piezothermoelastic composite cylindrical shell," Composite Structures, vol. 59, no. 1, pp. 45-60, 2003.

[14] R. Kadoli and N. Ganesan, "Free vibration and buckling analysis of composite cylindrical shells conveying hot fluid," Composite Structures, vol. 60, no. 1, pp. 19-32, 2003.

[15] M. Hemmatnezhad, R. Ansari, and M. Darvizeh, "Prediction of vibrational behavior of composite cylindrical shells under various boundary conditions," Applied Composite Materials, vol. 17, no. 2, pp. 225-241, 2010.

[16] N. Jaunky, N. F. Knight Jr., and D. R. Ambur, "Optimal design of general stiffened composite circular cylinders for global buckling with strength constraints," Composite Structures, vol. 41, no. 3-4, pp. 243-252, 1998.

[17] J. E. Helms, G. Li, and B. H. Smith, "Analysis of grid stiffened cylinders," in Proceedings of the Engineering Technology Conference on Energy (ETCE '01), pp. 247-251, Houstan, Tex, USA, February 2001.

[18] S. Black, "A grid stiffened alternative to cored laminates," HighPerformance Composites, vol. 10, pp. 48-51, 2004.

[19] N. Jaunky, N. F. Knight, and D. R. Ambur, "Formulation of an improved smeared stiffener theory of buckling analysis of gridstiffened composite panels," NASA Technical Memorandum 110162, 1995.

[20] J. L. Phillips and Z. Gurdal, "Structural analysis and optimum design of geodesically stiffened composite panels," NASA Report CCMS-90-05, 1990.

[21] G. Gerdon and Z. Gurdal, "Optimal design of geodesically stiffened composite cylindrical shells," AIAA Journal, vol. 23, no. 11, pp. 1753-1761, 1985.

[22] N. Jaunky, N. F. Knight Jr., and D. R. Ambur, "Optimal design of grid-stiffened composite panels using global and local buckling analyses," Journal of Aircraft, vol. 35, no. 3, pp. 478-486, 1998.

[23] J. T.-S. Wang and T.-M. Hsu, "Discrete analysis of stiffened composite cylindrical shells," AIAA Journal, vol. 23, no. 11, pp. 1753-1761, 1985.

[24] S. Kidane, G. Li, J. Helms, S. Pang, and E. Woldesenbet, "Buckling load analysis of grid stiffened composite cylinders," Composites B: Engineering, vol. 34, no. 1, pp. 1-9, 2003.

[25] E. Wodesenbet, S. Kidane, and S. Pang, "Optimization for buckling loads of grid stiffened composite panels," Composite Structures, vol. 60, no. 2, pp. 159-169, 2003.

[26] M. Yazdani, H. Rahimi, A. A. Khatibi, and S. Hamzeh, "An experimental investigation into the buckling of GFRP stiffened shells under axial loading," Scientific Research and Essays, vol. 4, no. 9, pp. 914-920, 2009.
[27] M. Yazdani and G. H. Rahimi, "The effects of helical ribs number and grid types on the buckling of thin-walled GFRPstiffened shells under axial loading," Journal of Reinforced Plastics and Composites, vol. 29, no. 17, pp. 2568-2575, 2010.

[28] M. Yazdani and G. H. Rahimi, "The behavior of GFRPstiffened and -unstiffened shells under cyclic axial loading and unloading," Journal of Reinforced Plastics and Composites, vol. 30, no. 5, pp. 440-445, 2011.

[29] G. H. Rahimi, M. Zandi, and S. F. Rasouli, "Analysis of the effect of stiffener profile on buckling strength in composite isogrid stiffened shell under axial loading," Aerospace Science and Technology, vol. 24, no. 1, pp. 198-203, 2013.

[30] S. Shi, Z. Sun, M. Ren, H. Chen, and X. Hu, "Buckling response of advanced grid stiffened carbon-fiber composite cylindrical shells with reinforced cutouts," Composites Part B: Engineering, vol. 44, no. 1, pp. 26-33, 2013.

[31] S. Shi, Z. Sun, M. Ren, H. Chen, and X. Hu, "Buckling resistance of grid-stiffened carbon-fiber thin-shell structures," Composites B: Engineering, vol. 45, no. 1, pp. 888-896, 2013.

[32] M. Hemmatnezhad, G. H. Rahimi, and R. Ansari, "On the free vibrations of grid-stiffened composite cylindrical shells," Acta Mechanica, vol. 225, no. 2, pp. 609-623, 2014.

[33] J. L. Sanders, "An improved first approximation theory for thin shells," NASA Report 24, 1959. 

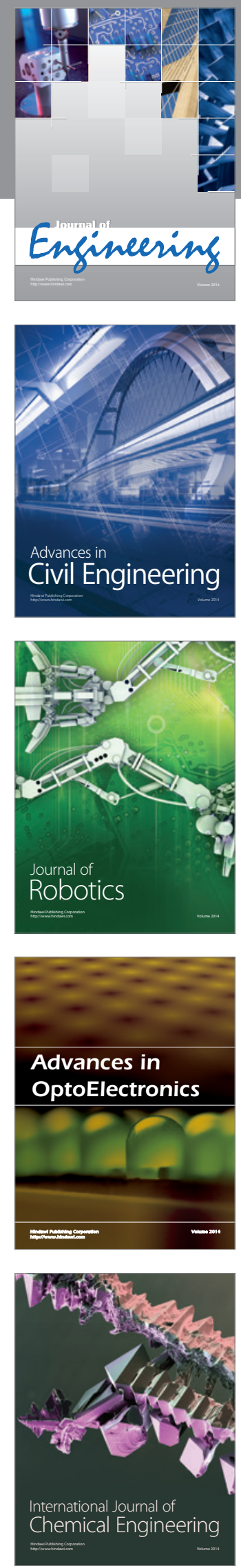

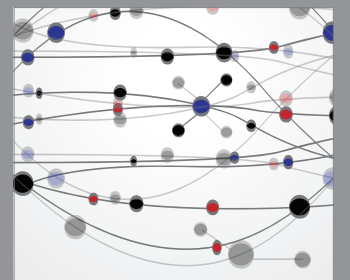

The Scientific World Journal
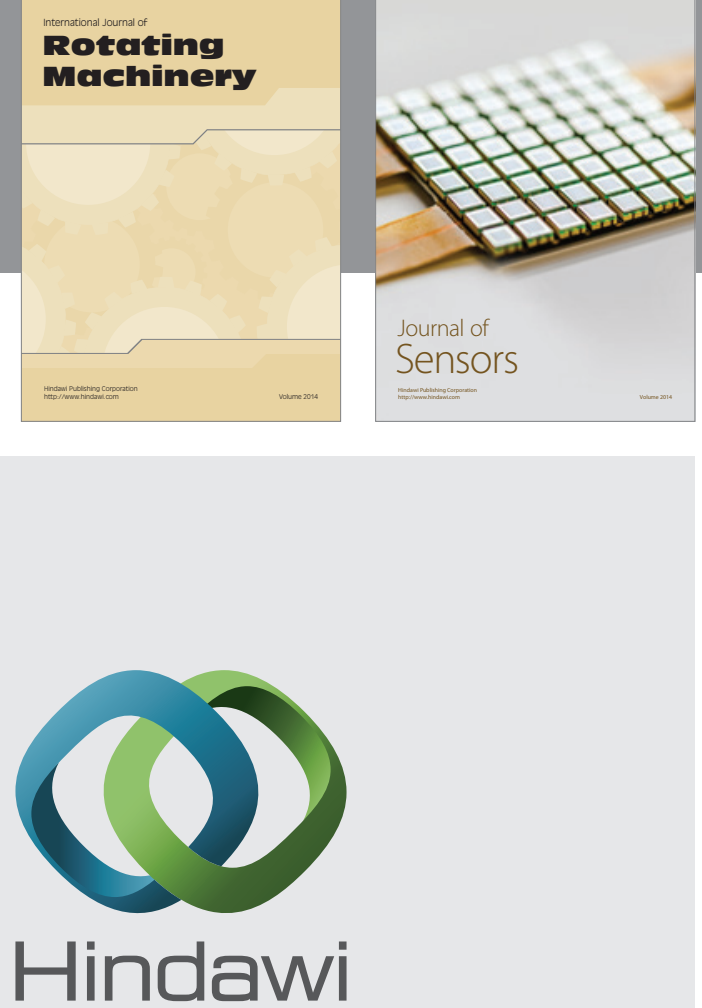

Submit your manuscripts at http://www.hindawi.com
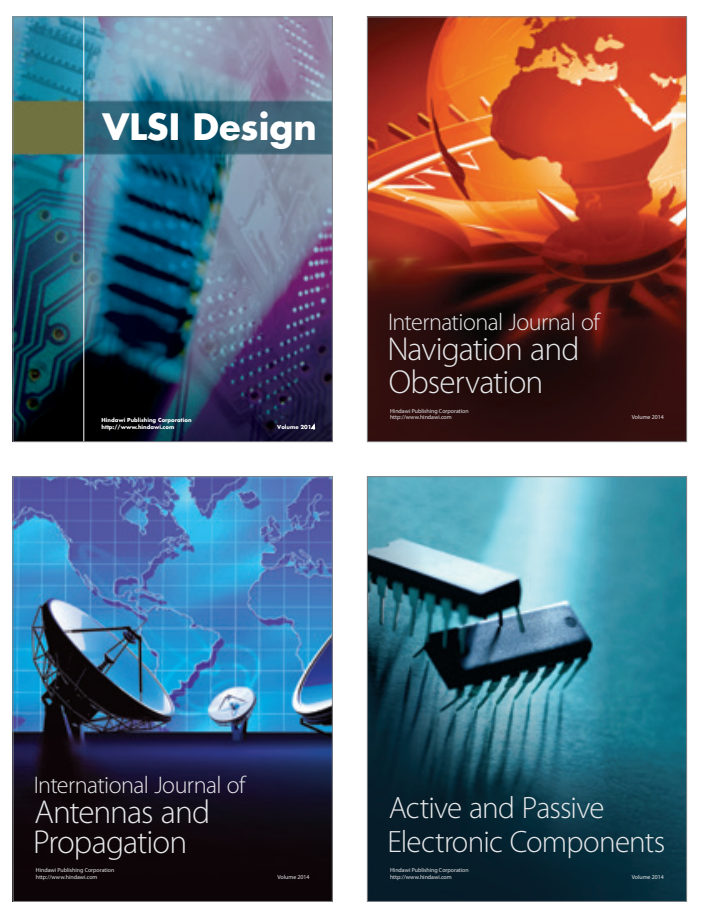
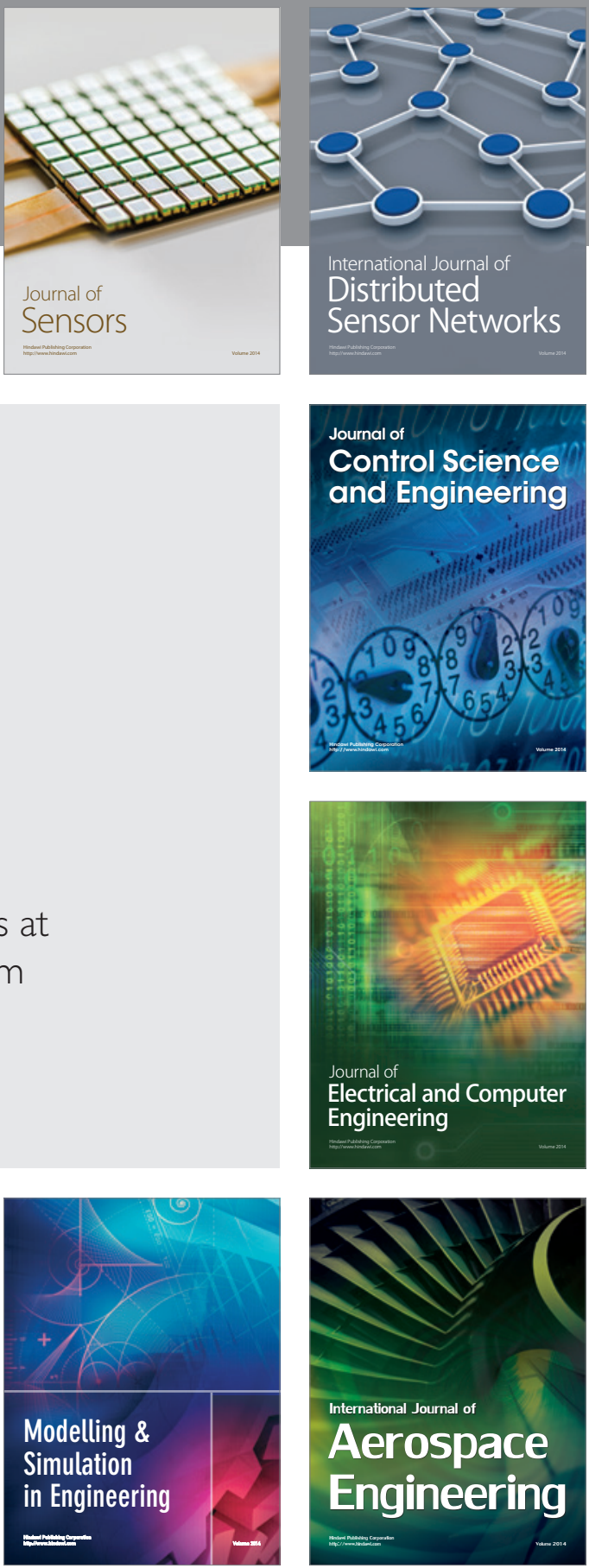

Journal of

Control Science

and Engineering
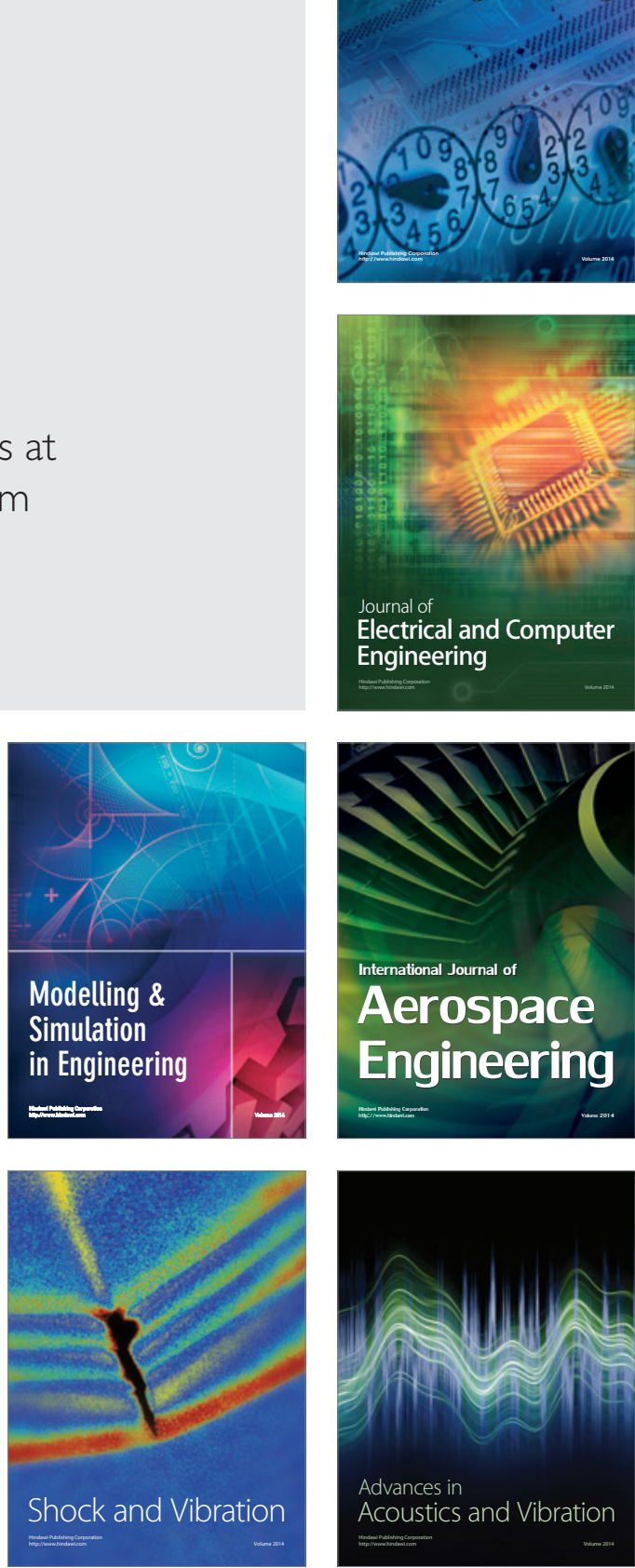